\title{
Detecting the presence of a singleton involves focal attention
}

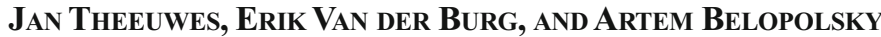 \\ Vrije Universiteit Amsterdam, Amsterdam, The Netherlands
}

\begin{abstract}
It has been claimed that the detection of a feature singleton can be based on activity in a feature map that allows coarse coding that something unique is present in the visual field. In the present study, participants detected the presence or absence of a color singleton. Even though the letter form of the color singleton was task-irrelevant, we showed that repeating the letter form of the singleton resulted in repetition priming on the next trial. Such repetition priming was not found when a nonsingleton letter was repeated as the singleton. Since the letter form of the color singleton could only be picked up by focal attention, the repetition priming effect indicates that focal attention is allocated to the feature singleton even in the simplest present-absent feature detection tasks. We showed that this effect is equally strong in conditions of low and high perceptual load. These results are inconsistent with theories that it is possible to detect a feature singleton without directing some form of attention to its location.
\end{abstract}

It is generally assumed that in visual perception the visual world is initially decomposed into maps consisting of simple features such as orientation, color, and luminance. Most theories of attention assume that simple features are coded independently and in parallel across the visual field (e.g., Treisman \& Gelade, 1980; Wolfe, 1994). Only when features of a particular object need to be combined, or when an object needs to be localized, must spatial attention be focused on that particular object (Treisman, 1988).

It has been argued that when detecting a feature singleton, participants can check a pooled response from the relevant feature map for the presence of activity anywhere in that map (e.g., Treisman, 1988). For example, Müller, Reimann, and Krummenacher (2003) argued that a manual detection response to a pop-out target can be released without focal attention. These notions indicate that it is possible to report the presence of a pop-out element without focal attention.

Others have argued that before a response (even the simplest pop-out detection response) can be made, spatial attention needs to be directed to the location of the feature singleton (Joseph, Chun, \& Nakayama, 1997; Nothdurft, 1999; Theeuwes, 1992; Theeuwes, Reimann, \& Mortier, 2006; Wolfe, 1994). According to this notion, postselective processing is obligatory for a detection response and occurs even in tasks in which identifying the target is not necessary. Therefore, even when a task requires participants to respond "present" to any odd-man-out target, it is assumed that a shift of spatial attention is necessary before a response can be given. For example, Joseph et al. (1997) showed that when participants were engaged in an attention-demanding central task that involved the detection of a target in a rapid stream of nontargets, they were unable to detect the presence of a pop-out feature at the periphery; because the central task required all attention, there was none left for detecting the pop-out feature. This study showed that attention was necessary for detecting a simple pop-out feature. Similar conclusions were reached in attentional blink studies in which observers had to detect the presence or absence of a single target, which was typically the second of two targets presented in a rapid stream of distractor letters (e.g., Raymond, Shapiro, \& Arnell, 1992). These studies also indicated that attention is needed for detection. Using a different approach, Theeuwes, Kramer, and Atchley (1999) showed that spatial attention played a role in the detection of a pop-out target. If no attention were needed for pop-out detection, the allocation of attention within the visual field should play no role in target detection. However, Theeuwes et al. (1999) showed that in a location cuing task, the allocation of attention in visual space had a large effect on the speed with which participants were able to detect the presence of a feature singleton.

From a neurophysiological perspective, it has been argued that attention is only required when ambiguities in neural coding have to be resolved (see, e.g., Luck \& Ford, 1998). When multiple objects fall inside the relatively large receptive fields of the ventral object recognition pathway, feature-specific neurons may respond, but because multiple objects are present inside the receptive field, it is unclear to which object the neurons have responded. Focusing attention on one object may resolve this ambiguity, because the neurons become responsive only to the features of the one object that is receiving

J.Theeuwes, j.theeuwes@psy.vu.nl 
attention (see, e.g., Luck, Girelli, McDermott, \& Ford, 1997). Luck and Ford provided evidence for this notion by showing that attention is necessary for discriminating conjunctions but not for detecting features. As an index for attentional allocation, Luck and Ford used the N2pc component of the ERP waveform. This is a negative-going deflection that is observable in the ERPs recorded over the posterior scalp contralateral to the attended side, roughly $175-300$ msec poststimulus. The N2pc is often thought to reflect the attentional selection of an item in a search array via the suppression of surrounding items. Luck and Ford showed a clear N2pc when participants performed a color-orientation discrimination. However, when participants only had to decide whether a particular color was present in the display, no N2pc was observed, especially when participants had to conduct a centrally demanding additional task. According to Luck and Ford, the absence of the N2pc indicates that attention is not needed in feature search to generate a present-absent response.

As shown by Luck and Ford (1998), when answering the question of whether attention is necessary for feature detection, one has to create conditions in which observers do not simply direct attention to the feature singleton because excess perceptual resources are available. Indeed, the perceptual load theory of Lavie (1995) suggests that with low perceptual load, task-irrelevant stimuli may receive excess attentional resources, allowing the processing of stimuli that are basically irrelevant for the task (see also Theeuwes, Kramer, \& Belopolsky, 2004; Yantis \& Johnston, 1990). In other words, in conditions of low perceptual load, attention may be shifted to the feature singleton not because attention is necessary for detection, but simply because excess resources allow participants to do so. However, in conditions of high perceptual load, there is not enough capacity to process irrelevant stimuli, and therefore task-irrelevant stimuli should not be processed. In line with this reasoning, Luck and Ford showed a small but significant $\mathrm{N} 2 \mathrm{pc}$ when participants had to perform a feature detection task. However, when this feature detection task was combined with a central attention-demanding task, the small, reliable N2pc disappeared, confirming the idea that under conditions of high load, participants can detect a feature singleton without shifting attention to its location. Obviously, Luck and Ford's central task required enough "resources" to discourage participants from shifting attention to the pop-out target, as revealed by the absence of an N2pc.

\section{EXPERIMENT 1}

In Experiment 1, participants had to detect the presence or absence of a single red element among gray elements. Even though letter form was completely irrelevant to the task, the red and gray elements consisted of alphanumeric characters. We examined intertrial effects. If it is possible to detect and respond to a feature singleton without directing attention to its location, then one would not expect the identity of the letter to have any effect on the next trial. However, if a feature detection response can only be given after a shift of spatial attention, one would expect that the identity of the completely irrelevant alphanumeric character would have an effect on the next trial. We combined this feature detection task with the same central task used by Luck and Ford (1998), in order to create conditions of high perceptual load. If a shift of spatial attention to the feature singleton is mandatory, then one would expect to find the same intertrial repetition priming effect regardless of the perceptual load.

\section{Method}

Participants. Ten students ( 9 male, 1 female; mean age 20.8 years, range 19-23 years) participated in the experiment as paid volunteers.

Stimuli. A trial started with the presentation of a white fixation dot $\left(85.8 \mathrm{~cd} / \mathrm{m}^{2}\right)$ at the center of the display for a period of $1,000 \mathrm{msec}$. The search display consisted of 10 random gray letters from the alphabet $\left(1.1^{\circ} \times 1.3^{\circ}\right.$, Courier New, $\left.21.6 \mathrm{~cd} / \mathrm{m}^{2}\right)$, equally spaced around the center of the computer screen on an imaginary circle with a radius of $5.3^{\circ}$. In half of the trials, one of the letters was red $\left(15.6 \mathrm{~cd} / \mathrm{m}^{2}\right)$, constituting the singleton target. When a feature singleton was present on both trials $n-1$ and $n$, there was a $50 \%$ chance that its identity would be repeated. Overall, on any given trial, the probability that the identity of a feature singleton would be repeated was $12.5 \%$. Simultaneously with the search display, a white degraded character was presented $\left(1.1^{\circ} \times 1.3^{\circ}\right.$, Courier New, $85.8 \mathrm{~cd} / \mathrm{m}^{2}$ ) in the center of the display. The character was randomly selected from the digits $1-8$. The total presentation time of the peripheral and central displays was $120 \mathrm{msec}$. See Figure 1.

Design and Procedure. Participants performed either a single or a dual task. In the single-task condition, participants were instructed to respond to the presence or absence of a singleton presented at the periphery and to ignore the digit. In the dual-task condition, participants were asked to respond to the singleton and at the same time to identify the central digit. The digit identification task was nonspeeded. Participants were required to give the response after performing the speeded search task.

In order to minimize ceiling effects in the digit task, we used the same adjustment procedure as Luck and Ford (1998). The amount of degradation was adjusted online by increasing or decreasing the number of pixels covering the digit, such that participants remained near $85 \%$ correct. If accuracy dropped below $85 \%$, the number of pixels was decreased. If accuracy was better than $85 \%$, the number of pixels was increased.

Participants received 10 experimental blocks ( 5 single-task and 5 dual-task) of 64 trials each, preceded by 2 practice blocks. Task blocks were counterbalanced and presented in alternating order. After each block, participants received feedback about their performance in the single- and dual-task conditions.

\section{Results}

Overall, performance in the digit task was at $85.9 \%$ correct, indicating that the adjustment worked adequately.

Trials in which participants responded faster than $300 \mathrm{msec}$ or slower than $1,400 \mathrm{msec}$ were excluded from further analysis. This led to a loss of $2.7 \%$ of the trials. Figure 2 presents mean correct reaction times (RTs) and errors as a function of target presence and task. Error rates were relatively low and were not analyzed further.

An ANOVA revealed no significant main effect of target presence $[F(1,9)=4.3, p=.06]$. The main effect of task was highly significant $[F(1,9)=18.2, p<.005]$, showing that participants responded more slowly to the presence or absence of the target singleton in dual-task conditions 


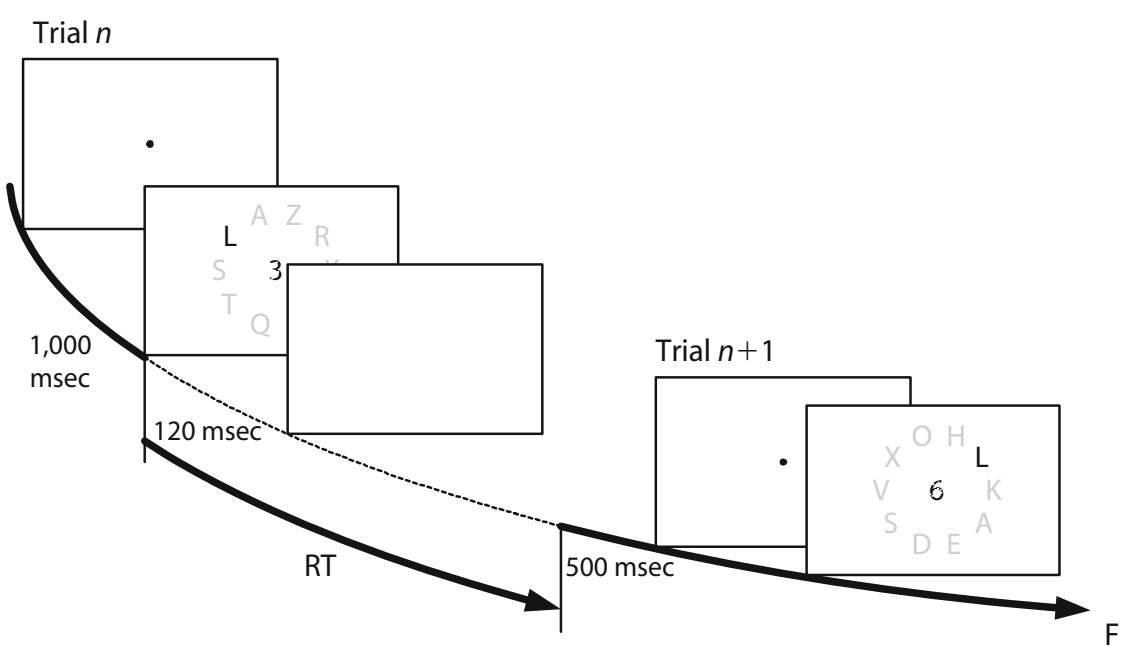

Figure 1. Example of a trial. A red target singleton was present on half of the trials. Participants made a speeded response to the presence or absence of the feature singleton target. The identity of the target (which was irrelevant for the task) was either repeated or not repeated on the next trial. In the single-task condition, participants only performed the speeded present-absent feature detection task; in the dual-task condition, they performed both the speeded feature detection task and a nonspeeded central letter identification task.

(614 msec) than in single-task conditions $(429 \mathrm{msec})$. The two-way interaction between task and target presence failed to reach significance $(F<1)$.

For target-present trials, we analyzed the effect of repeating the identity of the singleton in single- and dualtask conditions. The results are shown in Figure 3. Trials in which the digit was identified incorrectly were excluded from the analyses. There was a significant main effect of task $[F(1,9)=17.2, p<.005]$. There was also a main effect of target repetition $[F(1,9)=7.4, p=.023]$, indicating that participants were faster when the identity of the singleton was the same as on the previous trial $(497 \mathrm{msec})$ than when it was different $(515 \mathrm{msec})$. More importantly, the two-way interaction between task and target repetition was not even close to being significant $(F<1)$, indicating that the effect of repeating the target was the same in single- and dual-task conditions.

\section{Discussion}

The reliable effect of target repetition suggests that a feature detection response on trial $n$ was speeded when the singleton letter in trial $n-1$ happened to be identical. Note that the identity of the letters was completely irrelevant for the task. More importantly, this effect was the same in both low- and high-perceptual-load conditions. Indeed, there was no sign of an interaction $(F<1)$ between perceptual load (single vs. dual task) and target repetition. Note, however, that our load manipulation was successful. Relative to the single-task condition, search times increased by about $200 \mathrm{msec}$ when participants had to simultaneously perform the central digit identification and the peripheral detection task. These findings indicate that when detecting the presence of a feature singleton, attention was directed at its location, making the identity of the letter available and affecting the feature detection response on the next trial.

\section{EXPERIMENT 2}

Our interpretation of the present findings rests on the assumption that one can only obtain repetition priming after focal attention has been directed to the location of the pop-out target. However, if all letters are processed in parallel, one would also expect some degree of priming by the identity of nonsingleton letters. In the target-present trials of Experiment 2, either the letter constituting the singleton was repeated as a singleton on the next trial (as in Experiment 1), or a nonsingleton letter was repeated as the singleton, or the singleton was not a repeated letter (see the right panel of Figure 4 for examples). If repetition priming only occurs for the singleton, we would expect to find repetition priming only when the identity of the singleton was repeated (as in Experiment 1). However, if nonsingleton letters can also generate repetition priming, we would expect to find priming as well when a nonsingleton letter was repeated as a singleton on the next trial. In addition, even though the overall probability that the identity of the singleton would be repeated on the next trial was only $12.5 \%$, we reduced this probability even further, to $8.5 \%$, so that it was unlikely that participants would strategically "choose" to process the singleton's identity.

\section{Method}

Fifteen new students ( 4 male, 11 female; mean age 20.7 years, range 17-23 years) participated. The experiment was the same as the single-task condition of Experiment 1, except that in $50 \%$ of the target-present trials, a nonsingleton letter was repeated as a singleton on the next trial. The participants received 10 experimental blocks of 96 trials each, preceded by 1 practice block.

\section{Results}

Trials in which participants responded faster than $200 \mathrm{msec}$ or slower than $600 \mathrm{msec}$ were excluded from fur- 


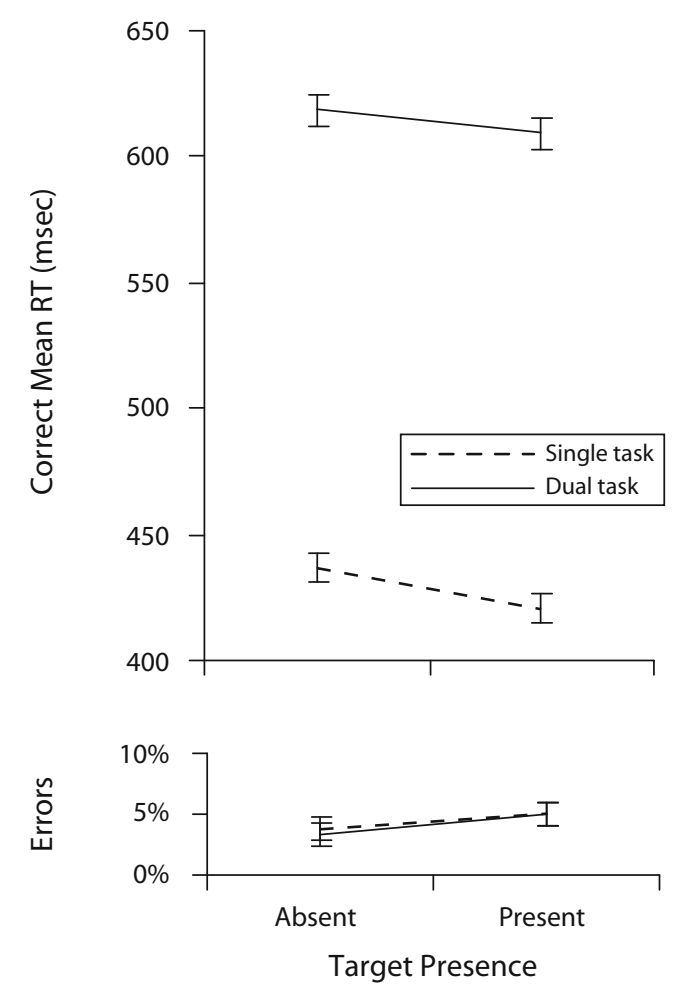

Figure 2. Experiment 1: Mean reaction times (RTs) and error percentages for singleton-present and -absent responses in singleand dual-task conditions. The error bars represent the .95 confidence intervals for within-subjects designs.

ther analysis. This led to a loss of $3.0 \%$ of the trials. Overall, error rates were relatively low (5.8\%) and were not analyzed further. There was no significant main effect of target presence $[t(14)=0.6, p=.535]$.

In the target-present condition, there were three conditions: singleton repetition, nonsingleton repetition, and no repetition. As is clear from Figure 4, as in Experiment 1, there was a reliable effect of target repetition $[F(2,28)=$ $5.2, p=.01]$.

Subsequent $t$ tests showed no difference between nonsingleton repetition and no-repetition trials $[t(14)=$ $0.798, p=.438]$, suggesting that nonsingletons did not generate an identity-priming effect. However, when a singleton was repeated, participants were significantly faster $(395 \mathrm{msec})$ than when a nonsingleton was repeated $[403 \mathrm{msec} ; t(14)=2.5, p=.028]$ and when there was no repetition [405 msec; $t(14)=2.7, p=.015$ ]. The results suggest that repetition priming only occurs when the identity of the singleton is repeated, and not when the identity of one of the nonsingletons is repeated.

\section{Discussion}

Experiment 2 showed that it is only possible to obtain repetition priming when focal attention is directed to the feature singleton. Without focal attention, one does not obtain repetition priming. When the identity of a nonsingleton was repeated on the next trial as a feature singleton, there was no speeding of the response. Our Experiment 2 confirmed our claim that the identity of a letter only be- comes available, causing repetition priming, after focal attention has been directed to the location.

\section{GENERAL DISCUSSION}

The present findings indicate that detecting the presence or absence of a single "pop-out" target requires spatial attention. The feature detection response was speeded when the identity of the singleton was identical to that on the previous trial, regardless of the perceptual load of the task. Experiment 2 showed that this identity priming did not occur when the identity of a nonsingleton was repeated as a singleton, suggesting that attention is needed for identity processing. Therefore, we conclude that even for the detection of the presence of a simple feature, spatial attention has to be directed to that feature's location.

One could argue that attention was directed to the location of the feature singleton not because it was necessary for the response but because participants chose to do so. If the central task did not produce enough load, there could have been enough time for participants to voluntarily direct attention to the location of the singleton, and this may have generated the identity-priming effect. There are several reasons why this alternative explanation is unlikely. First, our central task did produce high load, eliciting an increase in RT of about $200 \mathrm{msec}$ in the dual-task condition. If participants had directed attention voluntarily to the singleton, one would expect that in high-
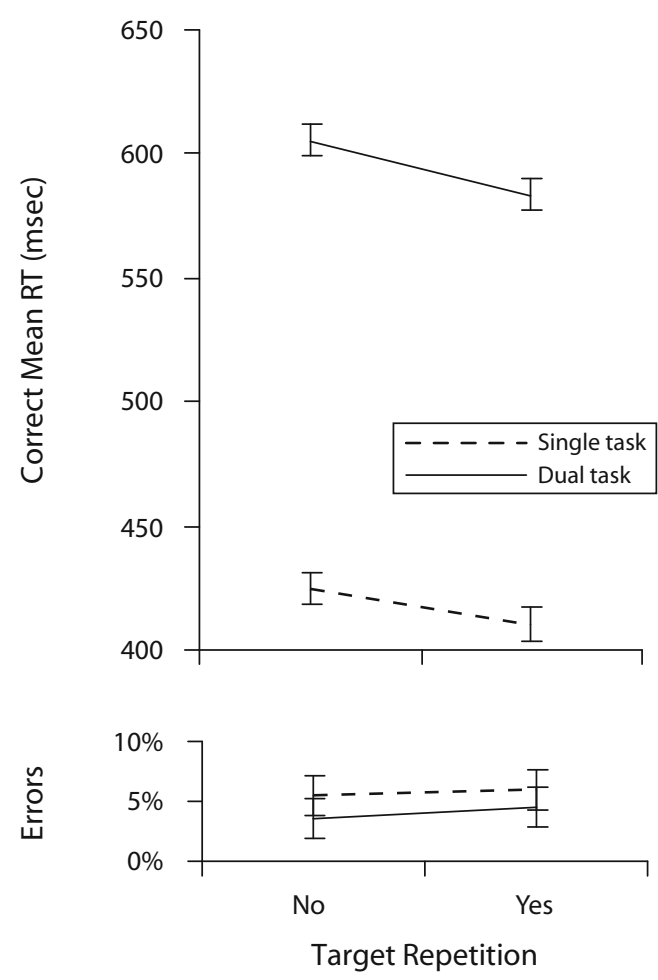

Figure 3. Experiment 1: Mean reaction times (RTs) and error percentages for conditions in which the identity of the singleton was either repeated or not repeated in single- and dual-task conditions. The error bars represent the $\mathbf{9 5}$ confidence intervals for within-subjects designs. 

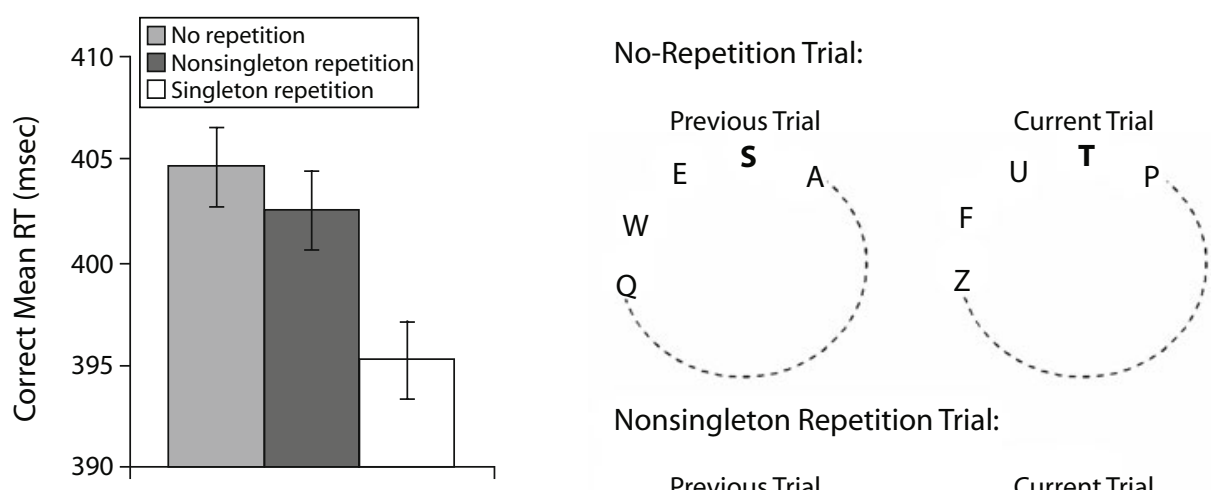

Nonsingleton Repetition Trial:
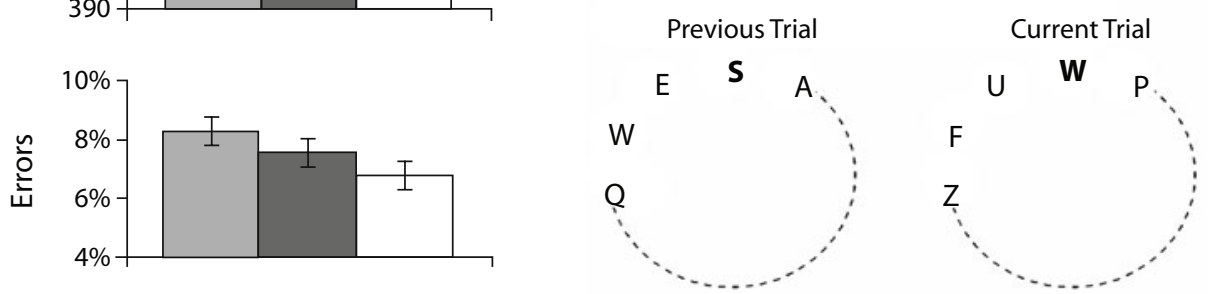

Singleton Repetition Trial:

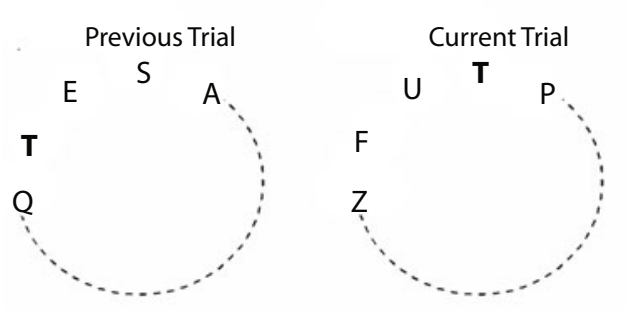

Figure 4. Experiment 2: Mean reaction times (RTs) and error percentages as a function of target repetition. As in Experiment 1, in a subset of target-present trials the letter constituting the singleton was repeated as a singleton on the next trial. In the remaining target-present trials, either the identity of the target singleton was identical to that of a nonsingleton letter on the previous trial (see the right panel of the figure for an example of nonsingleton repetition; here, the nonsingleton letter "W" in the previous trial matches the singleton target on the next trial) or the identity of the singleton did not match any of the letters on the previous trial (no-repetition trials). The error bars represent the .95 confidence intervals for within-subjects designs.

load conditions this voluntary shifting of attention would occur less often (at least in fewer trials) than in low-load conditions. In a high-load condition there is less time, because two tasks have to be performed simultaneously. Our results show no modulation of the identity-priming effect by high- and low-load conditions, suggesting that shifting attention to the location of the singleton is not a choice made by the participant, but a requirement of performing the task. Second, we used the very same central task as Luck and Ford (1998), and they showed that this task prevented participants from shifting attention to the feature singleton, as indexed by the absence of an N2pc. Third, according to the perceptual load theory of Lavie (1995), if processing resources are scarce (as in our high-load condition), all processing resources are limited to those operations that are absolutely necessary to accomplish the task. Again, in our experiment the magnitude of repetition priming was identical in low- and high-load conditions, suggesting that directing attention to the location was essential for accomplishing the task.
The present findings have important implications for theories of visual attention. According to the classic feature integration theory (FIT; Treisman \& Gelade, 1980) and the more modern version of FIT (Müller et al., 2003), when participants need to detect a single feature singleton, they can check a pooled response from the relevant feature map for the presence of activity anywhere in that map. Detecting a pop-out target thus should not require the involvement of focal attention. The present findings do not put in question the notion that separate and independent feature maps record the presence of features, but our findings do challenge the idea that a feature presenceabsence response can be generated without the involvement of attention.

Our findings appear to be inconsistent with the notion that attention is only required when ambiguities in neural coding have to be resolved (see, e.g., Luck et al., 1997). If attention is only necessary when multiple objects are present inside the same receptive field, there should have been no involvement of attention in this case, because there 
were no ambiguities. In our experiments, only one unique object was present, and this object was coded through unique activity within a color feature map. Participants only had to decide whether something unique was present in the visual field, not which color it was or its location. Obviously, from a neural-coding point of view, there were no ambiguities and therefore should have been no need for focal attention.

However, one could still claim that attention is not necessary for the detection of a singleton but only for the making of an overt response. The detection could occur without attention, but attention would be needed to consolidate the item into visual short-term memory (VSTM) in order to generate an overt response. For example, it has been argued that in attentional-blink-like tasks (see, e.g., Joseph et al., 1997), the feature singleton is fully identified, but the central task prevents storage of the feature singleton in VSTM. The observed impairment in feature detection thus is not due to a failure to detect the feature singleton per se, but to an impairment of postperceptual attentional processing (see, e.g., Vogel, Luck, \& Shapiro, 1998). Even though theoretically possible, this view basically implies that even though detection itself may occur without attention, responding to the feature (even in a simple feature detection) does require attention. This implies that attention operates at a postperceptual rather than a perceptual stage. Even though such an interpretation is feasible, it should be realized that even if one assumes that attention operates at a postperceptual stage, our repetition priming data show that this type of postperceptual attention allows the identification of complex letter features. Therefore, it seems reasonable to assume that attention is not just postperceptual, but operates at the level of perception as well.

The present findings appear inconsistent with Luck and Ford's (1998) claim that no attention is necessary for the detection of a simple feature. When their participants performed a central attentional demanding task similar to the one we used, simultaneously with a feature detection task, Luck and Ford observed no N2pc. Note, however, that this conclusion is only valid if it is assumed that the absence of an N2pc implies no attentional selection. It is possible that some type of attentional selection may occur that does not result in an N2pc. Indeed, it has been suggested that the N2pc reflects attentional selection of an item via the suppression of surrounding items (Luck \& Hillyard, 1994). Because in feature search there are no competing distracting elements near the target, there is no need for any suppression, and therefore an N2pc may not show up. However, this does not necessarily imply that spatial attention is not shifted to the location of the singleton.

In summary, our study shows that focal attention is required in order to perform the simplest feature detection task. This result is in line with the assumption that feature identification cannot be accomplished without the involvement of a perceptual-level attention mechanism (see, e.g., Joseph et al., 1997; Theeuwes, 1992).

\section{AUTHOR NOTE}

This research was funded by a grant from the Human Frontier Science Program (HSFP-RGP39/2005) to J.T. We thank Steve Franconeri, Chip Folk, and Steve Luck for comments on an earlier version. Correspondence concerning this article should be addressed to J. Theeuwes, Department of Cognitive Psychology, Vrije Universiteit Amsterdam, van der Boechorststraat 1, 1081 BT Amsterdam, The Netherlands (e-mail: j.theeuwes@psy.vu.nl).

\section{REFERENCES}

Joseph, J. S., Chun, M. M., \& Nakayama, K. (1997). Attentional requirements in a "preattentive" feature search task. Nature, 387, 805-807.

Lavie, N. (1995). Perceptual load as a necessary condition for selective attention. Journal of Experimental Psychology: Human Perception \& Performance, 21, 451-468.

LUCK, S. J., \& FORD, M. A. (1998). On the role of selective attention in visual perception. Proceedings of the National Academy of Sciences, 95, 825-830.

Luck, S. J., Girelli, M., McDermott, M. T., \& Ford, M. A. (1997). Bridging the gap between monkey neurophysiology and human perception: An ambiguity resolution theory of visual selective attention. Cognitive Psychology, 33, 64-87.

LuCK, S. J., \& Hillyard, S. A. (1994). Spatial filtering during visual search: Evidence from human electrophysiology. Journal of Experimental Psychology: Human Perception \& Performance, 20, 1000-1014.

Müller, H. J., Reimann, B., \& Krummenacher, J. (2003). Visual search for singleton feature targets across dimensions: Stimulusand expectancy-driven effects in dimensional weighting. Journal of Experimental Psychology: Human Perception \& Performance, 29, 1021-1035.

NothdURFT, H.-C. (1999). Focal attention in visual search. Vision Research, 39, 2305-2310.

Raymond, J. E., Shapiro, K. L., \& Arnell, K. M. (1992). Temporary suppression of visual processing in an RSVP task: An attentional blink? Journal of Experimental Psychology: Human Perception \& Performance, 18, 849-860.

Theeuwes, J. (1992). Perceptual selectivity for color and form. Perception \& Psychophysics, 51, 599-606.

Theeuwes, J., Kramer, A. F., \& Atchley, P. (1999). Attentional effects on preattentive vision: Spatial precues affect the detection of simple features. Journal of Experimental Psychology: Human Perception \& Performance, 25, 341-347.

Theeuwes, J., Kramer, A. F., \& Belopolsky, A. V. (2004). Attentional set interacts with perceptual load in visual search. Psychonomic Bulletin \& Review, 11, 697-702.

Theeuwes, J., Reimann, B., \& Mortier, K. (2006). Visual search for featural singletons: No top-down modulation, only bottom-up priming. Visual Cognition, 14, 466-489.

Treisman, A. [M.] (1988). Features and objects: The fourteenth Bartlett Memorial Lecture. Quarterly Journal of Experimental Psychology, 40A, 201-237.

Treisman, A. M., \& Gelade, G. (1980). A feature-integration theory of attention. Cognitive Psychology, 12, 97-136.

Vogel, E. K., LucK, S. J., \& ShapIRo, K. L. (1998). Electrophysiological evidence for a postperceptual locus of suppression during the attentional blink. Journal of Experimental Psychology: Human Perception \& Performance, 24, 1656-1674.

Wolfe, J. M. (1994). Guided Search 2.0: A revised model of visual search. Psychonomic Bulletin \& Review, 1, 202-238.

Yantis, S., \& Johnston, J. C. (1990). On the locus of visual selection: Evidence from focused attention tasks. Journal of Experimental Psychology: Human Perception \& Performance, 16, 135-149.

(Manuscript received July 27, 2007; revision accepted for publication December 6, 2007.) 